\title{
On cross-intersecting families of set partitions
}

\author{
Cheng Yeaw $\mathrm{Ku} *$ \\ Department of Mathematics \\ National University of Singapore \\ Singapore 117543. \\ matkcy@nus.edu.sg
}

\author{
Kok Bin Wong \\ Institute of Mathematical Sciences \\ University of Malaya \\ 50603 Kuala Lumpur, Malaysia \\ kbwong@um.edu .my
}

Submitted: Mar 29, 2012; Accepted: Dec 8, 2012; Published: Dec 31, 2012

Mathematics Subject Classifications: 05D05

\begin{abstract}
Let $\mathcal{B}(n)$ denote the collection of all set partitions of $[n]$. Suppose $\mathcal{A}_{1}, \mathcal{A}_{2} \subseteq \mathcal{B}(n)$ are cross-intersecting i.e. for all $A_{1} \in \mathcal{A}_{1}$ and $A_{2} \in \mathcal{A}_{2}$, we have $A_{1} \cap A_{2} \neq \varnothing$. It is proved that for sufficiently large $n$,

$$
\left|\mathcal{A}_{1}\right|\left|\mathcal{A}_{2}\right| \leqslant B_{n-1}^{2}
$$

where $B_{n}$ is the $n$-th Bell number. Moreover, equality holds if and only if $\mathcal{A}_{1}=\mathcal{A}_{2}$ and $\mathcal{A}_{1}$ consists of all set partitions with a fixed singleton.
\end{abstract}

Keywords: cross-intersecting family, Erdős-Ko-Rado, set partitions

\section{Introduction}

\section{$1.1 \quad$ Finite sets}

Let $[n]=\{1, \ldots, n\}$ and $\left(\begin{array}{c}{[n]} \\ k\end{array}\right)$ denote the family of all $k$-subsets of $[n]$. A fundamental result in extremal combinatorial set theory is the Erdös-Ko-Rado theorem ([6], [7], [22]) which asserts that if a family $\mathcal{A} \subseteq\left(\begin{array}{c}{[n]} \\ k\end{array}\right)$ is t-intersecting (i.e. $|A \cap B| \geqslant t$ for any $A, B \in \mathcal{A}$ ), then $|\mathcal{A}| \leqslant\left(\begin{array}{l}n-t \\ k-t\end{array}\right)$ for $n \geqslant(k-t+1)(t+1)$. Recently, there are several Erdös-Ko-Rado type results (see $[2,4,5,9,11,13,15,17,20,21]$ ), most notably is the result of Ellis, Friedgut and Pilpel [5], which states that for sufficiently large $n$ depending on $t$, a $t$-intersecting family $\mathcal{A}$ of permutations has size at most $(n-t)$ !, with equality if and only if $\mathcal{A}$ is a coset of the stabilizer of $t$ points, thus settling an old conjecture of Deza and Frankl [3].

${ }^{*}$ corresponding author 
Let $\mathcal{A}_{i} \subseteq\left(\begin{array}{c}{[n]} \\ k_{i}\end{array}\right)$ for $i=1,2, \ldots, r$. We say that the families $\mathcal{A}_{1}, \mathcal{A}_{2}, \ldots, \mathcal{A}_{r}$ are $r$-cross $t$-intersecting if $\left|A_{1} \cap A_{2} \cap \cdots \cap A_{r}\right| \geqslant t$ holds for all $A_{i} \in \mathcal{A}_{i}$. When $t=1$, we will just say $r$-cross intersecting instead of $r$-cross 1-intersecting. Furthermore when $r=2$ and $t=1$, we will just say cross-intersecting instead of 2-cross intersecting. It has been shown by Frankl and Tokushige [8] that if $\mathcal{A}_{1}, \mathcal{A}_{2}, \ldots, \mathcal{A}_{r} \subseteq\left(\begin{array}{c}{[n]} \\ k\end{array}\right)$ are $r$-cross intersecting, then for $n \geqslant r k /(r-1)$,

$$
\prod_{i=1}^{r}\left|\mathcal{A}_{i}\right| \leqslant\left(\begin{array}{l}
n-1 \\
k-1
\end{array}\right)^{r}
$$

For differing values of $k$ 's, we have the following result.

Theorem 1.1 (Bey [1], Matsumoto and Tokushige [18], Pyber [19]). Let $\mathcal{A}_{1} \subseteq\left(\begin{array}{l}{[n]} \\ k_{1}\end{array}\right)$ and $\mathcal{A}_{2} \subseteq\left(\begin{array}{l}{[n]} \\ k_{2}\end{array}\right)$ be cross-intersecting. If $k_{1}, k_{2} \leqslant n / 2$, then

$$
\left|\mathcal{A}_{1}\right|\left|\mathcal{A}_{2}\right| \leqslant\left(\begin{array}{c}
n-1 \\
k_{1}-1
\end{array}\right)\left(\begin{array}{c}
n-1 \\
k_{2}-1
\end{array}\right) .
$$

Equality holds for $k_{1}+k_{2}<n$ if and only if $\mathcal{A}_{1}$ and $\mathcal{A}_{2}$ consist of all $k_{1}$-element resp. $k_{2}$-element sets containing a fixed element.

\subsection{Set partitions}

A set partition of $[n]$ is a collection of pairwise disjoint nonempty subsets (called blocks) of $[n]$ whose union is $[n]$. Let $\mathcal{B}(n)$ denote the family of all set partitions of $[n]$. It is well-known that the size of $\mathcal{B}(n)$ is the $n$-th Bell number, denoted by $B_{n}$. A block of size one is also known as a singleton. We denote the number of all set partitions of $[n]$ which are singleton-free (i.e. without any singleton) by $\tilde{B}_{n}$.

A family $\mathcal{A} \subseteq \mathcal{B}(n)$ is said to be $t$-intersecting if any two of its members have at least $t$ blocks in common. $\mathrm{Ku}$ and Renshaw [14, Theorem 1.7 and Theorem 1.8] proved the following analogue of the Erdős-Ko-Rado theorem for set partitions.

Theorem 1.2 (Ku-Renshaw). Suppose $\mathcal{A} \subseteq \mathcal{B}(n)$ is a t-intersecting family. Then, for $n \geqslant n_{0}(t)$,

$$
|\mathcal{A}| \leqslant B_{n-t},
$$

with equality if and only if $\mathcal{A}$ consists of all set partitions with $t$ fixed singletons.

Recently, $\mathrm{Ku}$ and Wong [16, Theorem 1.4] proved a generalization of Theorem 1.2, which is an analogue of the Hilton-Milner Theorem [10] for set partitions.

In this paper, we will prove the following analogue of Theorem 1.1 for set partitions.

Theorem 1.3. Let $\mathcal{A}_{1}, \mathcal{A}_{2} \subseteq \mathcal{B}(n)$ be cross-intersecting. Then, for $n \geqslant n_{0}$,

$$
\left|\mathcal{A}_{1}\right|\left|\mathcal{A}_{2}\right| \leqslant B_{n-1}^{2} .
$$

Moreover, equality holds if and only if $\mathcal{A}_{1}=\mathcal{A}_{2}$ and $\mathcal{A}_{1}$ consists of all set partitions with a fixed singleton. 


\section{Splitting operation}

In this section, we will prove some important results regarding the splitting operation for $r$-cross $t$-intersecting families of set partitions. These results are the 'cross' version of [14, Proposition 3.1, 3.2, 3.3, 3.4].

Let $i, j \in[n], i \neq j$, and $P \in \mathcal{B}(n)$. Denote by $P_{[i]}$ the block of $P$ which contains $i$. We define the $(i, j)$-split of $P$ to be the following set partition:

$$
s_{i j}(P)= \begin{cases}P \backslash\left\{P_{[i]}\right\} \cup\left\{\{i\}, P_{[i]} \backslash\{i\}\right\} & \text { if } j \in P_{[i]}, \\ P & \text { otherwise. }\end{cases}
$$

For a family $\mathcal{A} \subseteq \mathcal{B}(n)$, let $s_{i j}(\mathcal{A})=\left\{s_{i j}(P): P \in \mathcal{A}\right\}$. Any family $\mathcal{A}$ of set partitions can be decomposed with respect to given $i, j \in[n]$ as follows:

$$
\mathcal{A}=\left(\mathcal{A} \backslash \mathcal{A}_{i j}\right) \cup \mathcal{A}_{i j},
$$

where $\mathcal{A}_{i j}=\left\{P \in \mathcal{A}: s_{i j}(P) \notin \mathcal{A}\right\}$. Define the $(i, j)$-splitting of $\mathcal{A}$ to be the family

$$
S_{i j}(\mathcal{A})=\left(\mathcal{A} \backslash \mathcal{A}_{i j}\right) \cup s_{i j}\left(\mathcal{A}_{i j}\right)
$$

It is not hard to see that $\left|S_{i j}(\mathcal{A})\right|=|\mathcal{A}|$.

Let $I(n, r, t)$ denote the set of all $r$-cross $t$-intersecting families of set partitions of $[n]$. Let $\mathbf{A}=\left\{\mathcal{A}_{1}, \mathcal{A}_{2}, \ldots, \mathcal{A}_{r}\right\} \in I(n, r, t)$. We set

$$
S_{i j}(\mathbf{A})=\left\{S_{i j}\left(\mathcal{A}_{1}\right), S_{i j}\left(\mathcal{A}_{2}\right), \ldots, S_{i j}\left(\mathcal{A}_{r}\right)\right\}
$$

and write $S_{i j}(\mathbf{A})=\mathbf{A}$ if $S_{i j}\left(\mathcal{A}_{l}\right)=\mathcal{A}_{l}$ for $l=1,2, \ldots, r$.

We define $|\mathbf{A}|=\prod_{l=1}^{r}\left|\mathcal{A}_{l}\right|$. It is not hard to see that

$$
|\mathbf{A}|=\prod_{i=1}^{r}\left|S_{i j}\left(\mathcal{A}_{l}\right)\right|
$$

An element $\mathbf{A}=\left\{\mathcal{A}_{1}, \mathcal{A}_{2}, \ldots, \mathcal{A}_{r}\right\} \in I(n, r, t)$ is said to be trivial, if $\mathcal{A}_{1}=\mathcal{A}_{2}=\cdots=$ $\mathcal{A}_{r}$ and $\mathcal{A}_{1}$ consists of all set partitions containing $t$ fixed singletons.

Proposition 2.1. Let $i, j \in[n], i \neq j$. If $\mathbf{A} \in I(n, r, t)$, then $S_{i j}(\mathbf{A}) \in I(n, r, t)$.

Proof. Let $\mathbf{A}=\left\{\mathcal{A}_{1}, \mathcal{A}_{2}, \ldots, \mathcal{A}_{r}\right\}$. For each $l=1,2, \ldots, r$, choose an $A_{l} \in S_{i j}\left(\mathcal{A}_{l}\right)$. If $A_{l} \in \mathcal{A}_{l}$ for all $l$, then $\left|A_{1} \cap A_{2} \cap \cdots \cap A_{r}\right| \geqslant t$. Without loss of generality, suppose $A_{l} \in s_{i j}\left(\left(\mathcal{A}_{l}\right)_{i j}\right)$ for $l=1, \ldots, q$, and $A_{l} \in \mathcal{A}_{l} \backslash\left(\mathcal{A}_{l}\right)_{i j}$ for $l=q+1, \ldots, r$. Then $A_{l}=s_{i j}\left(P_{l}\right)$ for $l=1, \ldots, q$, where $P_{l} \in\left(\mathcal{A}_{l}\right)_{i j} \subseteq A_{l}$.

Now there are at least $t$ blocks, say $M_{1}, M_{2}, \ldots, M_{t}$, that are all contained in $P_{1} \cap$ $\cdots \cap P_{q} \cap A_{q+1} \cap \cdots \cap A_{r}$. If $\{i, j\} \nsubseteq M_{y}$ for $y=1, \ldots, t$, then $M_{1}, M_{2}, \ldots, M_{t} \in A_{l}$ for $l=1, \ldots, q$. This implies that $M_{1}, M_{2}, \ldots, M_{t}$ are contained in $A_{1} \cap \cdots \cap A_{q} \cap A_{q+1} \cap \cdots \cap A_{r}$, and thus $\left|A_{1} \cap \cdots \cap A_{r}\right| \geqslant t$.

Suppose one of the $M_{y}$ contains $\{i, j\}$. We may assume that $\{i, j\} \subseteq M_{1}$. If $q=r$, then $\{i\}, M_{2}, \ldots, M_{t}$ are contained in $A_{1} \cap \cdots \cap A_{r}$, and thus $\left|A_{1} \cap \cdots \cap A_{r}\right| \geqslant t$. Suppose 
$1 \leqslant q<r$. Since $A_{l} \in \mathcal{A}_{l} \backslash\left(\mathcal{A}_{l}\right)_{i j}$ for $l \geqslant q+1$, we must have $s_{i j}\left(A_{l}\right) \in \mathcal{A}_{l}$. Note that $M_{2}, \ldots, M_{t}$ are contained in $P_{1} \cap \cdots \cap P_{q} \cap s_{i j}\left(A_{q+1}\right) \cap \cdots \cap s_{i j}\left(A_{r}\right)$. Since $\mid P_{1} \cap \cdots \cap$ $P_{q} \cap s_{i j}\left(A_{q+1}\right) \cap \cdots \cap s_{i j}\left(A_{r}\right) \mid \geqslant t$, there is a block $M_{t+1}$ disjoint from $M_{1}, M_{2}, \ldots, M_{t}$, that is contained in $P_{1} \cap \cdots \cap P_{q} \cap s_{i j}\left(A_{q+1}\right) \cap \cdots \cap s_{i j}\left(A_{r}\right)$. Now $M_{t+1}$ is a block in $A_{1} \cap \cdots \cap A_{q} \cap A_{q+1} \cap \cdots \cap A_{r}$, for $\{i, j\} \nsubseteq M_{t+1}$. Hence $\left|A_{1} \cap \cdots \cap A_{r}\right| \geqslant t$.

Proposition 2.2. Let $n \geqslant t+1$. Suppose $\mathbf{A} \in I(n, r, t)$ and $|\mathbf{A}|>1$. Let $i, j \in[n], i \neq j$. If $S_{i j}(\mathbf{A})$ is trivial, then $\mathbf{A}$ is trivial.

Proof. Let $\mathbf{A}=\left\{\mathcal{A}_{1}, \mathcal{A}_{2}, \ldots, \mathcal{A}_{r}\right\}$. Then $S_{i j}(\mathbf{A})=\left\{S_{i j}\left(\mathcal{A}_{1}\right), S_{i j}\left(\mathcal{A}_{2}\right), \ldots, S_{i j}\left(\mathcal{A}_{r}\right)\right\}$ and by Proposition 2.1, $S_{i j}(\mathbf{A}) \in I(n, r, t)$. Since $S_{i j}(\mathbf{A})$ is trivial, $S_{i j}\left(\mathcal{A}_{1}\right)=S_{i j}\left(\mathcal{A}_{2}\right)=$ $\cdots=S_{i j}\left(\mathcal{A}_{r}\right)$ and $S_{i j}\left(\mathcal{A}_{1}\right)$ consists of all set partitions containing $t$ fixed singletons, say $\left\{x_{1}\right\},\left\{x_{2}\right\}, \ldots,\left\{x_{t}\right\}$. Note that $T=\left\{\left\{x_{1}\right\},\left\{x_{2}\right\}, \ldots,\left\{x_{t}\right\},[n] \backslash\left\{x_{1}, \ldots, x_{t}\right\}\right\} \in S_{i j}\left(\mathcal{A}_{1}\right)$. If $T \in s_{i j}\left(\left(\mathcal{A}_{1}\right)_{i j}\right)$, then $T=s_{i j}(P)$ for a $P \in\left(\mathcal{A}_{1}\right)_{i j} \subseteq \mathcal{A}_{1}$. Note that $P$ will have exactly $t$ blocks. Now, if $Q_{l} \in \mathcal{A}_{l}$ for $l=2, \ldots, r$, then $P=Q_{2}=\cdots=Q_{r}$, for $\left|P \cap Q_{2} \cap \cdots \cap Q_{r}\right| \geqslant t$. Therefore $\mathcal{A}_{2}=\mathcal{A}_{3}=\cdots=\mathcal{A}_{r}=\{P\}$, and this implies that $\mathcal{A}_{1}=\{P\}$. So $|\mathbf{A}|=\prod_{l=1}^{r}\left|\mathcal{A}_{l}\right|=1$, a contradiction. So we may assume that $T \in \mathcal{A}_{1} \backslash\left(\mathcal{A}_{1}\right)_{i j} \subseteq \mathcal{A}_{1}$. Similarly, $T \in \mathcal{A}_{2} \cap \cdots \cap \mathcal{A}_{r}$.

Suppose $\mathcal{A}_{1} \neq S_{i j}\left(\mathcal{A}_{1}\right)$. Then there is a $P \in \mathcal{A}_{1}$ with $s_{i j}(P) \notin \mathcal{A}_{1}$. Now

$$
|P \cap \overbrace{T \cap \cdots \cap T}^{r-1}| \geqslant t,
$$

for $T \in \mathcal{A}_{2} \cap \cdots \cap \mathcal{A}_{r}$. Suppose $[n] \backslash\left\{x_{1}, \ldots, x_{t}\right\}$ is a block in $P$. Since $T$ has exactly $t+1$ blocks, we deduce that $P=T$. This means that $T \in\left(\mathcal{A}_{1}\right)_{i j}$, and $s_{i j}(T) \in S_{i j}\left(\mathcal{A}_{1}\right)$. So $T \notin S_{i j}\left(\mathcal{A}_{1}\right)$, a contradiction.

Suppose $[n] \backslash\left\{x_{1}, \ldots, x_{t}\right\}$ is not a block in $P$. Then $\left\{x_{1}\right\},\left\{x_{2}\right\}, \ldots,\left\{x_{t}\right\}$ are blocks in $P$. This implies that $P \in S_{i j}\left(\mathcal{A}_{1}\right)$, for $S_{i j}(\mathbf{A})$ is trivial. Since $P \in \mathcal{A}_{1}$, we must have $s_{i j}(P) \in \mathcal{A}_{1}$, a contradiction. Hence $\mathcal{A}_{1}=S_{i j}\left(\mathcal{A}_{1}\right)$. Similarly $\mathcal{A}_{l}=S_{i j}\left(\mathcal{A}_{l}\right)$ for $l=2, \ldots, r$.

An element $\mathbf{A} \in I(n, r, t)$ is said to be compressed if for any $i, j \in[n], i \neq j$, we have $S_{i j}(\mathbf{A})=\mathbf{A}$. For a set partition $P$, let $\sigma(P)=\{x:\{x\} \in P\}$ denote the union of its singletons (block of size 1). For a family $\mathcal{A}$ of set partitions, let $\sigma(\mathcal{A})=\{\sigma(P): P \in$ $\mathcal{A}\}$. Note that $\sigma(\mathcal{A})$ is a family of subsets of $[n]$. Now for $\mathbf{A}=\left\{\mathcal{A}_{1}, \ldots, \mathcal{A}_{r}\right\}$, where $\mathcal{A}_{1}, \ldots, \mathcal{A}_{r} \subseteq \mathcal{B}(n)$, set $\sigma(\mathbf{A})=\left\{\sigma\left(\mathcal{A}_{1}\right), \ldots, \sigma\left(\mathcal{A}_{r}\right)\right\}$. We say $\sigma(\mathbf{A})$ is $r$-cross $t$-intersecting if $\sigma\left(\mathcal{A}_{1}\right), \ldots, \sigma\left(\mathcal{A}_{r}\right)$ are $r$-cross $t$-intersecting.

Proposition 2.3. Given an element $\mathbf{A} \in I(n, r, t)$, by repeatedly applying the splitting operations, we eventually obtain a compressed $\mathbf{A}^{*} \in I(n, r, t)$ with $\left|\mathbf{A}^{*}\right|=|\mathbf{A}|$.

Proof. Note that if $S_{i j}(\mathbf{A}) \neq \mathbf{A}$, then the $(i, j)$-splits of some partitions are finer than the originals and therefore will move down in the partition lattice. Eventually this results in a compressed family of partitions.

For a compressed $\mathbf{A}$, its $r$-cross $t$-intersecting property can be transferred to $\sigma(\mathbf{A})$, thus allowing us to access the structure of $\mathbf{A}$ via the structure of $\sigma(\mathbf{A})$. 
Proposition 2.4. If $\mathbf{A} \in I(n, r, t)$ is compressed, then $\sigma(\mathbf{A})$ is $r$-cross t-intersecting.

Proof. Let $\mathbf{A}=\left\{\mathcal{A}_{1}, \mathcal{A}_{2}, \ldots, \mathcal{A}_{r}\right\}$. Assume, for a contradiction, that there exist $P_{l} \in \mathcal{A}_{l}$, $l=1, \ldots, r$ such that $\left|\sigma\left(P_{1}\right) \cap \cdots \cap \sigma\left(P_{l}\right)\right|<t$. Since $\left|P_{1} \cap \cdots \cap P_{r}\right| \geqslant t$, there are $s \geqslant t-\left|\sigma\left(P_{1}\right) \cap \cdots \cap \sigma\left(P_{l}\right)\right|$ common blocks of $P_{1}, \ldots, P_{r}$ (each of size at least 2), say $M_{1}, \ldots, M_{s}$, which are disjoint from $\sigma\left(P_{1}\right) \cup \cdots \cup \sigma\left(P_{r}\right)$. Fix two distinct points $x_{e}, y_{e}$ from each $M_{e}$. Then $P_{1}^{*}=s_{x_{s} y_{s}}\left(\cdots\left(s_{x_{1} y_{1}}\left(P_{1}\right)\right) \cdots\right) \in \mathcal{A}_{1}$, for $\mathbf{A}$ is compressed. Now $\left|P_{1}^{*} \cap P_{2} \cap \cdots \cap P_{r}\right|<t$, a contradiction.

\section{Proof of main result}

Recall that the size of $\mathcal{B}(n)$ is the $n$-th Bell number, denoted by $B_{n}$, and the number of all set partitions of $[n]$ which are singleton-free (i.e. without any singleton) is denoted by $\tilde{B}_{n}$.

The following identities for $B_{n}$ and $\tilde{B}_{n}$ are straightforward.

Lemma 3.1. Let $n \geqslant 2$. Then

$$
\begin{aligned}
B_{n} & =\sum_{k=0}^{n}\left(\begin{array}{l}
n \\
k
\end{array}\right) \tilde{B}_{n-k}, \\
\tilde{B}_{n} & =\sum_{k=1}^{n-1}\left(\begin{array}{c}
n-1 \\
k
\end{array}\right) \tilde{B}_{n-1-k},
\end{aligned}
$$

with the conventions $B_{0}=\tilde{B}_{0}=1$.

Note in passing that $\tilde{B}_{1}=0$. By (1) and (2),

$$
B_{n}=\tilde{B}_{n}+\tilde{B}_{n+1} .
$$

Given a real number $x$, we shall denote the greatest integer less than or equal to $x$, by $\lfloor x\rfloor$. Note that $\lfloor x\rfloor \leqslant x<\lfloor x\rfloor+1$. Some useful inequalities involving $B_{n}$ can be found in $[12]$. However we just need the following inequality.

Lemma 3.2. There is a positive integer $n_{0}$ such that for $n \geqslant n_{0}$,

$$
\tilde{B}_{n-1}>8^{n} \sum_{\left\lfloor\frac{n}{2}\right\rfloor \leqslant k \leqslant n}\left(\begin{array}{l}
n \\
k
\end{array}\right) \tilde{B}_{n-k} .
$$

Proof. By (2),

$$
\begin{aligned}
\sum_{\left\lfloor\frac{n}{2}\right\rfloor \leqslant k \leqslant n}\left(\begin{array}{l}
n \\
k
\end{array}\right) \tilde{B}_{n-k} & \leqslant \tilde{B}_{n-\left\lfloor\frac{n}{2}\right\rfloor+2} \sum_{\left\lfloor\frac{n}{2}\right\rfloor \leqslant k \leqslant n}\left(\begin{array}{l}
n \\
k
\end{array}\right) \\
& \leqslant 2^{n} \tilde{B}_{n-\left\lfloor\frac{n}{2}\right\rfloor+2} .
\end{aligned}
$$


So it is sufficient to show that $\tilde{B}_{n-1} / \tilde{B}_{n-\left\lfloor\frac{n}{2}\right\rfloor+2}>(16)^{n}$.

Again by (2), for any fixed $q, \tilde{B}_{m} / \tilde{B}_{m-2}>q$ for sufficiently large $m$. Therefore

$$
\begin{aligned}
\frac{\tilde{B}_{n-1}}{\tilde{B}_{n-\left\lfloor\frac{n}{2}\right\rfloor+2}} & \geqslant\left(\frac{\tilde{B}_{n-\left\lfloor\frac{n}{2}\right\rfloor+2 u}}{\tilde{B}_{n-\left\lfloor\frac{n}{2}\right\rfloor+2 u-2}}\right) \cdots\left(\frac{\tilde{B}_{n-\left\lfloor\frac{n}{2}\right\rfloor+6}}{\tilde{B}_{n-\left\lfloor\frac{n}{2}\right\rfloor+4}}\right)\left(\frac{\tilde{B}_{n-\left\lfloor\frac{n}{2}\right\rfloor+4}}{\tilde{B}_{n-\left\lfloor\frac{n}{2}\right\rfloor+2}}\right) \\
& >q^{u-1},
\end{aligned}
$$

where $u=\left\lfloor\frac{1}{2}\left(\left\lfloor\frac{n}{2}\right\rfloor-3\right)\right\rfloor$. Clearly $u-1 \geqslant \frac{n}{8}$. So if we choose $q=(16)^{8}$, then for sufficiently large $n$, the lemma follows.

Let $\mathbf{A}=\left\{\mathcal{A}_{1}, \ldots, \mathcal{A}_{r}\right\} \in I(n, r, t)$ be compressed. We say $\sigma(\mathbf{A})$ is trivial if there is a fixed $t$-set, say $T$, such that $T \subseteq \sigma\left(P_{l}\right)$ for all $P_{l} \in \mathcal{A}_{l}, l=1, \ldots, r$.

Theorem 3.3. Let $\mathbf{A} \in I(n, 2,1)$ be compressed. If $\sigma(\mathbf{A})$ is non-trivial, then

$$
|\mathbf{A}|<B_{n-1}^{2} .
$$

Proof. Let $\mathbf{A}=\left\{\mathcal{A}_{1}, \mathcal{A}_{2}\right\}$. For $k \geqslant 1$, let $\mathcal{F}_{l k}=\sigma\left(\mathcal{A}_{l}\right) \cap\left(\begin{array}{c}{[n]} \\ k\end{array}\right)$. If $\mathcal{F}_{l 1} \neq \varnothing$ for $l=1,2$, then $\sigma(\mathbf{A})$ is trivial. So we may assume that $\mathcal{F}_{21}=\varnothing$. By Proposition 2.4, $\sigma(\mathbf{A})$ is cross-intersecting. Note that $\left|\mathcal{A}_{1}\right| \leqslant \sum_{1 \leqslant k \leqslant n}\left|F_{1 k}\right| \tilde{B}_{n-k}$ and $\left|\mathcal{A}_{2}\right| \leqslant \sum_{2 \leqslant k \leqslant n}\left|F_{2 k}\right| \tilde{B}_{n-k}$. Then

$$
\begin{aligned}
\left|\mathcal{A}_{1}\right| & \leqslant \sum_{1 \leqslant k<\left\lfloor\frac{n}{2}\right\rfloor}\left|F_{1 k}\right| \tilde{B}_{n-k}+\sum_{\left\lfloor\frac{n}{2}\right\rfloor \leqslant k \leqslant n}\left|F_{1 k}\right| \tilde{B}_{n-k} \\
& \leqslant \sum_{1 \leqslant k<\left\lfloor\frac{n}{2}\right\rfloor}\left|F_{1 k}\right| \tilde{B}_{n-k}+\sum_{\left\lfloor\frac{n}{2}\right\rfloor \leqslant k \leqslant n}\left(\begin{array}{l}
n \\
k
\end{array}\right) \tilde{B}_{n-k}
\end{aligned}
$$

and

$$
\left|\mathcal{A}_{2}\right| \leqslant \sum_{2 \leqslant k<\left\lfloor\frac{n}{2}\right\rfloor}\left|F_{2 k}\right| \tilde{B}_{n-k}+\sum_{\left\lfloor\frac{n}{2}\right\rfloor \leqslant k \leqslant n}\left(\begin{array}{l}
n \\
k
\end{array}\right) \tilde{B}_{n-k} .
$$

Let

$$
\begin{aligned}
Q & =\sum_{\left\lfloor\frac{n}{2}\right\rfloor \leqslant k \leqslant n}\left(\begin{array}{l}
n \\
k
\end{array}\right) \tilde{B}_{n-k} \\
M_{1} & =\sum_{1 \leqslant k<\left\lfloor\frac{n}{2}\right\rfloor}\left|F_{1 k}\right| \tilde{B}_{n-k} \\
M_{2} & =\sum_{2 \leqslant k<\left\lfloor\frac{n}{2}\right\rfloor}\left|F_{2 k}\right| \tilde{B}_{n-k} .
\end{aligned}
$$

Then

$$
\begin{aligned}
|\mathbf{A}| & \leqslant\left(M_{1}+Q\right)\left(M_{2}+Q\right) \\
& =M_{1} M_{2}+M_{1} Q+M_{2} Q+Q^{2} .
\end{aligned}
$$


Note that by (2) and (3),

$$
\begin{aligned}
M_{l} & \leqslant \tilde{B}_{n} \sum_{1 \leqslant k<\left\lfloor\frac{n}{2}\right\rfloor}\left|F_{l k}\right| \\
& \leqslant \tilde{B}_{n} \sum_{1 \leqslant k<\left\lfloor\frac{n}{2}\right\rfloor}\left(\begin{array}{l}
n \\
k
\end{array}\right) \\
& \leqslant 2^{n} \tilde{B}_{n} \\
& \leqslant 2^{n} B_{n-1} .
\end{aligned}
$$

By Lemma 3.2 and (3), $Q \leqslant \frac{1}{8^{n}} \tilde{B}_{n-1}<\frac{1}{8^{n}} B_{n-1}<B_{n-1}$. Therefore

$$
\begin{aligned}
M_{1} Q+M_{2} Q+Q^{2} & <\left(2^{n}+2^{n}+1\right) B_{n-1}\left(\frac{\tilde{B}_{n-1}}{8^{n}}\right) \\
& <\frac{1}{2} B_{n-1} \tilde{B}_{n-1} .
\end{aligned}
$$

By Theorem 1.1,

$$
\begin{aligned}
M_{1} M_{2} & \leqslant \sum_{\substack{1 \leqslant k_{1}<\left\lfloor\frac{n}{2}\right\rfloor \\
2 \leqslant k_{2}<\left\lfloor\frac{n}{2}\right\rfloor}}\left(\begin{array}{c}
n-1 \\
k_{1}-1
\end{array}\right)\left(\begin{array}{c}
n-1 \\
k_{2}-1
\end{array}\right) \tilde{B}_{n-k_{1}} \tilde{B}_{n-k_{2}} \\
& =\left(\sum_{1 \leqslant k<\left\lfloor\frac{n}{2}\right\rfloor}\left(\begin{array}{l}
n-1 \\
k-1
\end{array}\right) \tilde{B}_{n-k}\right)\left(\sum_{2 \leqslant k<\left\lfloor\frac{n}{2}\right\rfloor}\left(\begin{array}{l}
n-1 \\
k-1
\end{array}\right) \tilde{B}_{n-k}\right) .
\end{aligned}
$$

By (1),

$$
\begin{aligned}
& \sum_{1 \leqslant k<\left\lfloor\frac{n}{2}\right\rfloor}\left(\begin{array}{l}
n-1 \\
k-1
\end{array}\right) \tilde{B}_{n-k}<B_{n-1} \\
& \sum_{2 \leqslant k<\left\lfloor\frac{n}{2}\right\rfloor}\left(\begin{array}{l}
n-1 \\
k-1
\end{array}\right) \tilde{B}_{n-k}<B_{n-1}-\tilde{B}_{n-1} .
\end{aligned}
$$

So $M_{1} M_{2} \leqslant\left(B_{n-1}-\tilde{B}_{n-1}\right) B_{n-1}$, and

$$
\begin{aligned}
|\mathbf{A}| & <B_{n-1}^{2}-\tilde{B}_{n-1} B_{n-1}+\frac{1}{2}\left(B_{n-1}\right) \tilde{B}_{n-1} \\
& <B_{n-1}^{2} .
\end{aligned}
$$

\section{Proof of Theorem 1.3.}

Let $\mathbf{A} \in I(n, 2,1)$ be of maximum size. We may assume that $\mathbf{A}$ has size at least $B_{n-1}^{2}$. Repeatedly apply the splitting operations until we obtain an $\mathbf{A}^{*} \in I(n, 2,1)$ such that 
$\mathbf{A}^{*}$ is compressed (Proposition 2.3). By Proposition 2.4, $\sigma\left(\mathbf{A}^{*}\right)$ is cross-intersecting. If $\sigma\left(\mathbf{A}^{*}\right)$ non-trivial, by Theorem 3.3, $|\mathbf{A}|<B_{n-1}^{2}$, a contradiction. So $\sigma\left(\mathbf{A}^{*}\right)$ is trivial. This implies that $\mathbf{A}^{*}$ is trivial, for $\mathbf{A}$ is of maximum size. It then follows from Proposition 2.2 that $\mathbf{A}$ is trivial.

\section{Acknowledgement}

We would like to thank the anonymous referee for the comments that helped us make several improvements to this paper.

\section{References}

[1] C. Bey, On cross-intersecting families of sets, Graphs Combin. 21 (2005), 161-168.

[2] P. J. Cameron and C. Y. Ku, Intersecting families of permutations, European J. Combin. 24 (2003), 881-890.

[3] M. Deza and P. Frankl, On the maximum number of permutations with given maximal or minimal distance, J. Combin. Theory Ser. A 22 (1977), 352-360.

[4] D. Ellis, Stability for t-intersecting families of permutations, J. Combin. Theory Ser. A 118 (2011), 208-227.

[5] D. Ellis, E. Friedgut and H. Pilpel, Intersecting families of permutations, J. Amer. Math. Soc. 24 (2011), 649-682.

[6] P. Erdős, C. Ko and R. Rado, Intersection theorems for systems of finite sets, Quart. J. Math. Oxford Ser. 212 (1961), 313-318.

[7] P. Frankl, The Erdős-Ko-Rado theorem is true for $n=$ ckt, Col. Soc. Math. J. Bolyai 18 (1978), 365-375.

[8] P. Frankl and N. Tokushige, On $r$-cross intersecting families of sets, Combin. Probab. Comput. 20 (2011), 749-752.

[9] C. Godsil and K. Meagher, A new proof of the Erdős-Ko-Rado theorem for intersecting families of permutations, European J. Combin. 30 (2009), 404-414.

[10] A. J. W. Hilton and E. C. Milner, Some intersection theorems for systems of finite sets, Quart. J. Math. Oxford (2) 18 (1967), 369-384.

[11] G. Hurlbert and V. Kamat, Erdős-Ko-Rado theorems for chordal graphs and trees, J. Combin. Theory Ser. A 118 (2011), 829-841.

[12] M. Klazar, Counting set systems by weight, Electron. J. Combin. 12 (2005), \#R11.

[13] C. Y. Ku and I. Leader, An Erdős-Ko-Rado theorem for partial permutations, Discrete Math. 306 (2006), 74-86.

[14] C. Y. Ku and D. Renshaw, Erdős-Ko-Rado theorems for permutations and set partitions, J. Combin. Theory Ser. A 115 (2008), 1008-1020. 
[15] C. Y. Ku and T. W. H. Wong, Intersecting families in the alternating group and direct product of symmetric groups, Electron. J. Combin. 14 (2007), \#R25.

[16] C. Y. Ku and K. B. Wong, An Analogue of Hilton-Milner Theorem for Set Partitions, preprint available at arXiv: 1109.0417.

[17] B. Larose and C. Malvenuto, Stable sets of maximal size in Kneser-type graphs, European J. Combin. 25 (2004), 657-673.

[18] M. Matsumoto and N. Tokushige, The exact bound in the Erdős-Ko-Rado theorem for cross-intersecting families, J. Combin. Theory Ser. A 52 (1989), 90-97.

[19] L. Pyber, A new generalization of the Erdős-Ko-Rado theorem, J. Combin. Theory Ser. A 43 (1986), 85-90.

[20] N. Tokushige, A product version of the Erdős-Ko-Rado theorem, J. Combin. Theory Ser. A 118 (2011), 1575-1587.

[21] J. Wang and S. J. Zhang, An Erdős-Ko-Rado-type theorem in Coxeter groups, European J. Combin. 29 (2008), 1112-1115.

[22] R.M. Wilson, The exact bound in the Erdős-Ko-Rado theorem, Combinatorica 4 (1984), 247-257. 